\title{
Software Educativo como herramienta de apoyo para niños autistas
}

\section{Educational Software as a support tool for autistic children}

\author{
GÓMEZ-MANUEL, Esbeidy†*, DOMÍNGUEZ-CAMPOMANES, Margarita, KATT-MORALES, Luz \\ Alondra y GILBON-ABURTO, Antonio
}

Universidad Tecnológica del Sureste de Veracruz, Tecnologías de la Información

ID $1^{\text {er }}$ Autor: Esbeidy, Gómez-Manuel / ORC ID: 0000-0003-0765-3402, Researcher ID Thomson: G-2859-2019, arXiv Author ID: 2419916, CVU CONACYT ID: 599053

ID $1^{\text {er }}$ Coautor: Margarita, Dominguez-Campomanes / Researcher ID Thomson: G-5015-2019, arXiv Author ID: 2635670, CVU CONACYT ID: 947280

ID $2^{\text {do }}$ Coautor: Luz Alondra, Katt-Morales / ORC ID: 0000-0002-9982-8718, arXiv Author ID: 2417152, Researcher ID Thomson: S-6606-2018, CVU CONACYT ID: 412698

ID $3^{\text {er }}$ Coautor: Antonio, Gilbon-Aburto

DOI: $10.35429 / J A D .2019 .9 .3 .12 .19$

Recibido 22 de Junio, 2019; Aceptado 30 de Septiembre, 2019

\section{Resumen}

Vivimos en la era digital o era informática que ofrece nuevos dispositivos (computadoras, celulares inteligentes, y tabletas electrónicas) y Aplicaciones Electrónicas (APPs por sus siglas en inglés) con un enorme potencial para el tratamiento educativo de los niños con Trastorno de Espectro Autista (en adelante TEA) definido por el manual de psiquiatría DSM IV como "un trastorno neurobiológico del desarrollo que ya se manifiesta durante los tres primeros años de vida y que perdurará a lo largo de todo el ciclo vital". (Asociación Estadounidense de Psiquiatría, 2002). Tras el análisis de los escasos centros de atención en el sur de Veracruz para los niños con TEA y la facilidad que ofrecen las TIC's para crear APP's, surge el software "ELY" que se divide en tres apartados importantes: Actividades Mentales, Actividades sociales y Actividades de Interacción social. Estos apartados contienen actividades que permiten reforzar las habilidades cognitivas y sociales ausentes en los niños con TEA. Además, con esta herramienta los tutores no requirieron de constante inversión económica en materiales didácticos como tarjetas de cartón, lápices de colores, figuras geométricas, entre otros. Los participantes que usaron el software en su tratamiento mejoraron su reconocimiento de las emociones como felicidad, tristeza y enojo, también progresaron reconociendo patrones y colores. Esto comprueba que las Tecnologías de la Información y Comunicación (TIC's) en esta investigación apoyaron los procesos específicos de enseñanzaaprendizaje de niños con TEA, fomentando la comunicación, la socialización y la imaginación los cuales son factores esenciales para relacionarse con las personas que los rodean.

\begin{abstract}
We live in the digital age or computer age that offers new devices (computers, smart phones, and electronic tablets) and Electronic Applications (APPs) with enormous potential for the educational treatment of children with Autism Spectrum Disorder ( hereinafter ASD) defined by the DSM IV psychiatry manual as "a neurobiological developmental disorder that already manifests itself during the first three years of life and that will last throughout the entire life cycle." (US ASSOCIATION OF PSYCHIATRY, 2002 ) After the analysis of the few centers of attention in the south of Veracruz for children with ASD and the ease offered by ICTs to create PPPs, the "ELY" software emerges that is divided into three important sections: Mental Activities, Social Activities and Social interaction activities. These sections contain activities that reinforce absent cognitive and social skills in children with ASD. In addition, with this tool the tutors did not require constant economic investment in teaching materials such as cardboard cards, colored pencils, geometric figures, among others. Participants who used the software in their treatment improved their recognition of emotions such as happiness, sadness and anger, also progressed by recognizing patterns and colors. This proves that Information and Communication Technologies (ICTs) in this research supported the specific teaching-learning processes of children with ASD, promoting communication, socialization and imagination, which are essential factors for relating to people who surround.
\end{abstract} y Diseño. 2019, 3-9: 12-19

\footnotetext{
$\dagger$ Investigador contribuyendo como primer autor.
} 


\section{Objetivo General}

Diseñar y desarrollar un software administrativo integral orientado a centros de atención para niños con TEA que permita la gestión de pacientes, tutores, terapias y terapeutas, y que incluya actividades que promuevan la enseñanza-aprendizaje de habilidades cognitivas y sociales de los niños con autismo.

\section{Objetivos específicos}

- Investigar e indagar sobre el comportamiento de los niños autistas, mediante entrevistas y reuniones con personas especializadas en el campo del TEA, para definir las actividades que se incluirán en el sistema interactivo.

- Investigar e indagar sobre el comportamiento de los niños autistas

- Conocer la forma en que se trabaja con los niños en los centros de atención autista e interactuar conaquellos que hayan demostrado avance en su tratamiento.

- Desarrollar el sistema usando estándares de usabilidad que permitan obtener un software fácil de usar por los niños autistas y personal que los apoya.

- Implementar la metodología Scrum en el ciclo de vida del proyecto.

- Realizar pruebas al sistema para detectar y corregir posibles errores. Elaborar reportes finales. Área/s de aplicación.

Los centros de atención al no contar con herramientas digitales que les ayude a ofrecer una correcta administración de sus pacientes provocan deserción de los tratamientos, es por eso que ELY aporta beneficios importantes al apoyo del tratamiento del síndrome asperger que van desde tener un registro de las terapias que tiene el niño durante su estancia en el centro de atención al que acude hasta poder cotejar los resultados obtenidos en el uso de las actividades, con el comportamiento del niño su entorno.
Los tutores no requieren hacer una constante inversión económica en materiales didácticos (tarjetas de cartón, lápices de colores, figuras geométricas, entre otros).

Permite reforzar habilidades de comunicación verbal, socialización e imaginativas en menor tiempo, comparado con los tratamientos que emplean materiales didácticos como: tarjetas de cartón, lápices de colores, figuras geométricas, entre otros.

ELY es un software educativo como herramientas de apoyo para el tratamiento del trastorno autista, automatizando y transformando actividades comunes en actividades que combinan las palabras con apoyos visuales, teniendo como principal objetivo el aprendizaje cognitivo y social de los niños con el síndrome de asperger. Las actividades están clasificadas en tres grandes grupos o apartados los cuales son: habilidad mental, habilidad social e interacción social.

Ofrece a los tutores y a los terapeutas de los niños llevar un registro del tiempo, aciertos, intentos y niveles que el niño obtiene durante el uso de ELY, esto permitirá saber con certeza el grado de avance que registra el niño.

Ely es una herramienta informática que dispone de módulos para los pacientes (niños autistas), tutores, terapeutas, y administradores. Cada módulo dispone de funciones específicas para cada tipo de usuario evitando así que usuarios malintencionados modifiquen o alteren datos importantes en el sistema.

Ely está dirigido a fundaciones o centros de atención que proporcionen tratamiento a niños con síndrome de Asperger que se encuentren viviendo dentro de la república mexicana, los cuales presenten avances reconociendo patrones, colores $\mathrm{y}$ emociones. Se guardara el registro de los siguientes aspectos: fechas de terapias, información profesional del terapeuta, información básica de los tutores y niños, así como los resultados que estos obtienen durante el uso de ELY. 


\section{Metodología}

La metodología utilizada en el desarrollo de esta investigación fue experimental utilizando la comparación de grupos para la comprobación de la relación entre las variables independientes y dependientes. Para valorar las habilidades sociales y cognitivas, antes y después del uso de ELY, se utilizaron tres escalas valorativas (comunicación verbal, relaciones sociales e imitación motriz) de tipo Likert con ítems, que se valoran del uno al cinco, donde uno significa que el niño presenta dificultad o no tiene adquirida la capacidad que se evalúa, y por el contrario, una puntuación de cinco, nos indica que no presenta dificultad o tiene adquirida dicha capacidad. Por lo tanto, cuanto mayor sea la puntuación, mayor será el grado de competencia social de la persona valorada. Los ítems de dichas escalas fueron evaluados previamente por psicólogos y terapeutas que participan en el tratamiento de los niños con TEA, quienes determinaron la validez del contenido.

ELY fue desarrollado utilizando la metodología de desarrollo Scrum, los beneficios que ésta nos proporcionó se mencionan a continuación:

\section{- Cumplimento de expectativas \\ - $\quad$ Mayor calidad del software \\ - Mayor productividad \\ - $\quad$ Reducción de riesgos}

Para la producción del software en la versión de escritorio se utilizaron las herramientas informáticas: Visual Studio, lenguaje $\mathrm{C \#}$, con el sistema gestor de base de datos MySQL y el editor de gráficos Photoshop. Las actividades que se realizaron durante el desarrollo fueron:

$\begin{array}{ll}- & \text { La reunión de planificación de Sprint } \\ - & \text { Scrums diarios } \\ - & \text { Trabajo de desarrollo } \\ - & \text { Revisión del Sprint } \\ - & \text { Retrospectiva del Sprint. }\end{array}$

Procedimiento de desarrollo de software

Requisitos: Para el análisis de requerimientos se utilizaron las siguientes técnicas: observación, entrevista y encuesta.
Diseño: Se tomaron en cuenta las actividades que los niños realizan en su terapia para trasladar esos escenarios educativos a una plataforma digital.

Codificación: En la codificación del software se empleó el lenguaje de programación orientado a objetos C\# y el IDE Visual Studio.

Pruebas: La fase de prueba se realizó en un periodo de dos semanas.

El diseño metodológico de esta investigación acerca del juego como estrategia didáctica, es de carácter cualitativo (descriptivo e interpretativo). Entendiéndolo como "multimetódico, naturalista e interpretativa. Es decir que los investigadores cualitativos indagan en situaciones naturales, intentando dar sentido o interpretar los fenómenos en los términos del significado que las personas les otorgan.

La investigación cualitativa abarca, el estudio, uso y recolección de una variedad de materiales empíricos que describen los momentos habituales y problemáticos y los significados en la vida de los individuos" (Vasilachis de Gialdino, 2009). [4]

Las herramientas de investigación utilizadas en este proyecto son:

Encuesta: De tipo exploratoria con cinco reactivos, a seis padres de niños con TEA, que asisten a la fundación Yanavi para darle continuidad a su tratamiento. La encuesta se contestó entre ocho a diez minutos.

Entrevista: Consta de diecisiete preguntas entre las que más destacan son: ¿Qué tipo de estrategias tienen para tratar al trastorno autista? , ¿A qué edad los niños autistas están más dispuestos a recibir una enseñanza virtual.

Uno de las principales fuentes de información sobre el tema de trastorno espectro autista es el Manual diagnóstico y estadístico de los trastornos mentales(DSM IV), así como artículos científicos que proponen herramientas que implementa las TIC como medio de enseñanzaaprendizaje en el tratamiento de niños con TEA. 
Se observó durante un periodo considerable las conductas de diez niños con TEA en un rango de edad de cinco a once años; poca interacción con las personas que los rodean, nula o escasa comunicación de emociones básicas (felicidad, tristeza, enojo) y escasa o nula comunicación verbal con las personas que lo rodean, son las conductas que se presentaron con más frecuencia en el periodo de tiempo de la observación.

Todas estas proporcionan información primaria del tratamiento y las actividades que realizan los niños con TEA.

Los centros de atención de niños con TEA no cuentan con un sistema de ayuda para administrar actividades como: llevar un control de los niños que asisten a terapias, tener registro de los terapeutas que laboran en la institución, contener las fechas de las terapias programadas para los pacientes, y almacenar información del familiar.

Los indicadores son: reconocimiento y percepción de emociones básicas (felicidad, tristeza y enojo), también patrones, mayor concentración y comprensión de las actividades que realizan.

Para realizar las pruebas se buscan las siguientes características en los niños:

- Haber sido diagnosticado con síndrome de asperger.

- $\quad$ Tener avances en su tratamiento.

- Reconocer colores y patrones

- $\quad$ El experimento se realizó con 15 niños de la zona sur de Veracruz, durante un periodo de cuarenta y cinco minutos por sesión, teniendo lugar dos sesiones por semana, a lo largo de dos meses.

- Para el análisis de la información se concentraron los resultados en tablas las cuales muestran los puntos que obtuvieron los niños antes y después del uso de ELY.

Se presenta un diagrama de cómo está estructurada la información dentro de la aplicación web ELY. Ver figura 1.

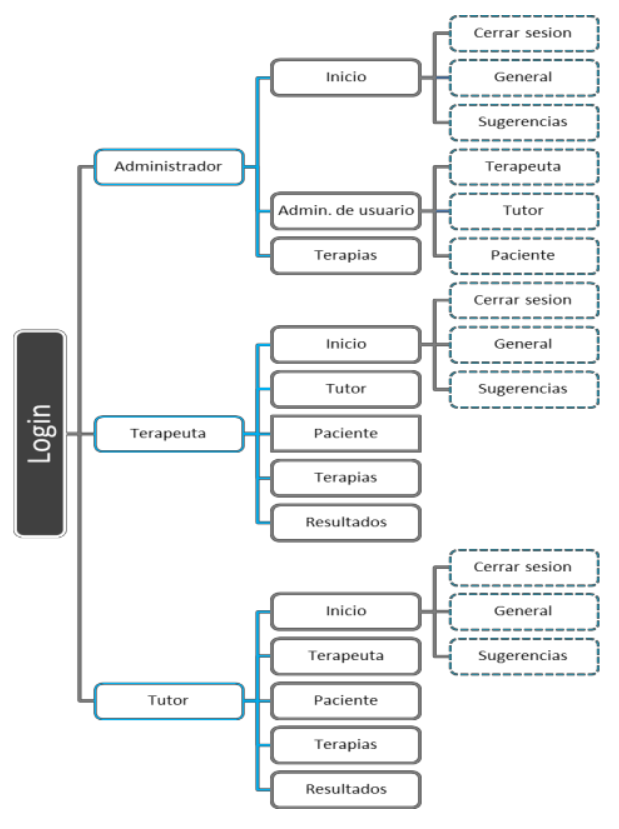

Figura 1 Estructura de la información página web (Elaboración Propia, 2019)

Las interfaces gráficas de los juegos desarrollados se describen a continuación. En la Figura 2 se muestra la pantalla del Juego del gato, en esta actividad se lleva el conteo del tiempo, intentos, errores y el nivel. El niño cuenta con nueve cartas a elegir entre los colores azul y café. El juego termina cuando se forme una línea de tres cartas de un solo color.

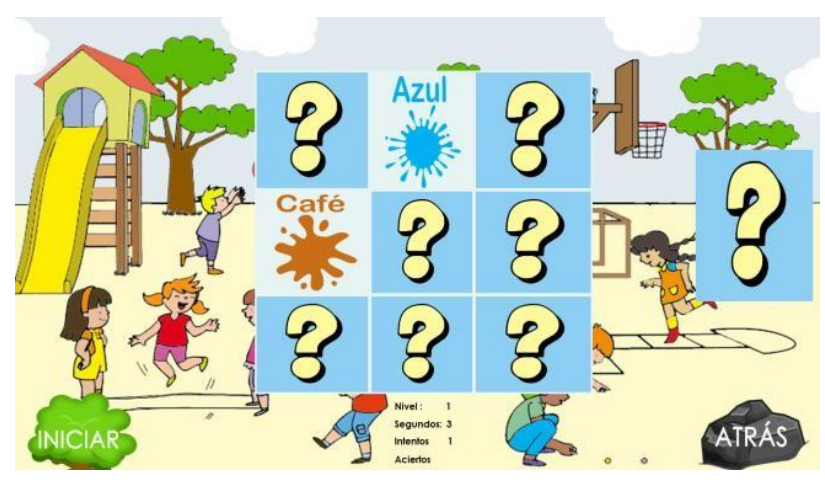

Figura 2 Juego del gato

(Elaboración Propia, 2019)

La interfaz del Juego de arrastrar y soltar figuras geométricas, esto permitirá a los niños identificar las formas comunes de su entorno. Figura 3. 


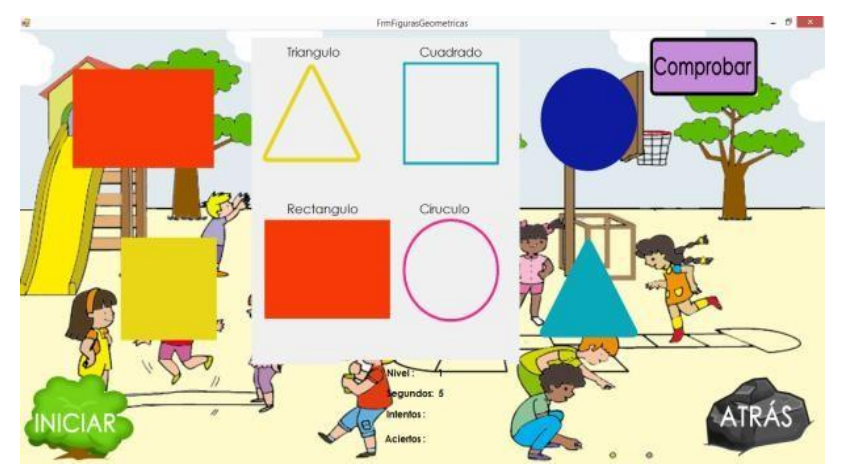

Figura 3 Juego de arrastrar y soltar (Elaboración Propia, 2019)

En la Figura 4 se muestra la pantalla del Juego de memorama para identificar las emociones, en esta actividad se lleva el control del tiempo, nivel, intentos y nivel que obtiene el niño autista. En este primer nivel el niño dispone de seis cartas con las emociones básicas (tristes, enojadas y felices). El juego se termina cuando se armen todos los pares de tarjetas.

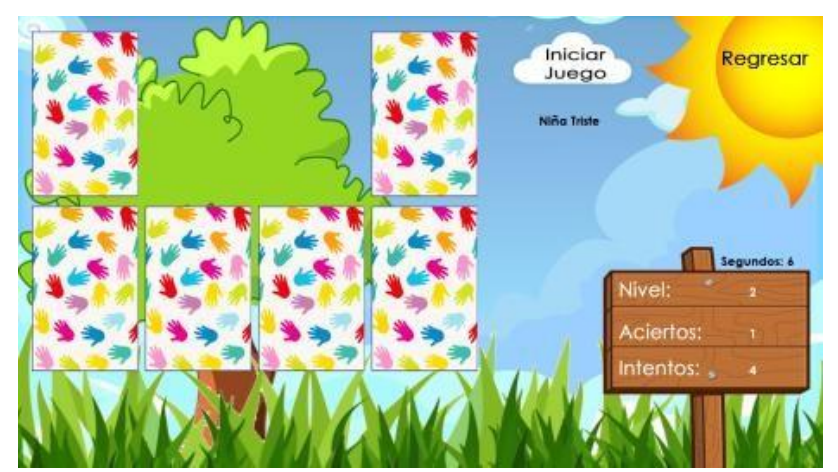

Figura 4 Juego de memorama

(Elaboración Propia, 2019)

Se visualiza el Juego del rompecabezas, donde el niño podrá reconocer la comunicación verbal por medio de una imagen de dos niños conversando. En este juego únicamente se toma en cuenta el tiempo empleado para resolverlo. Ver figura 5.

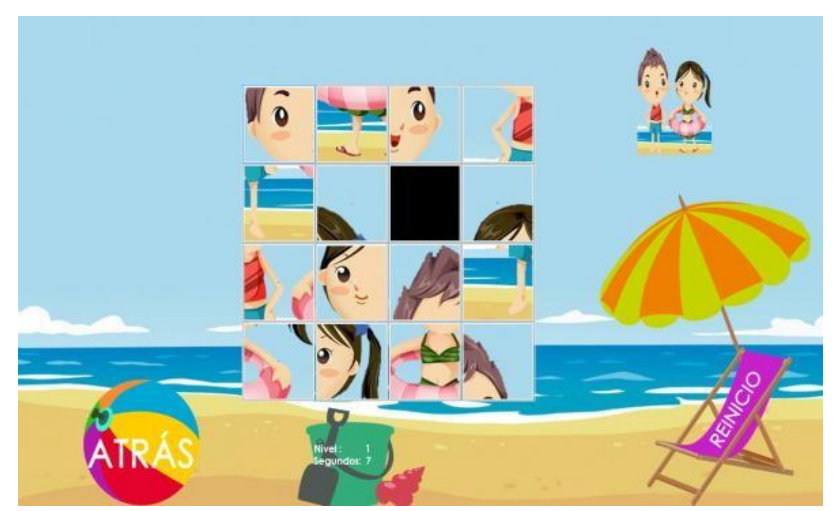

Figura 5 Juego del Rompecabezas

(Elaboración Propia, 2019)
La aplicación web permitirá usuario administrador pueda realizar altas, bajas revisión de $\mathrm{E} / \mathrm{S}$ de usuarios ya sea por la página web la aplicación misma. Los usuarios terapeutas y tutores solo tendrán acceso a la información de los pacientes pero no podrán realizar altas o bajas. En este apartado se muestra un calendario e información que el tutor solo visualiza. Una vez que el usuario ingresa al Sitio Web, lo primero que observa es la página principal del sitio. Ver figura 6.

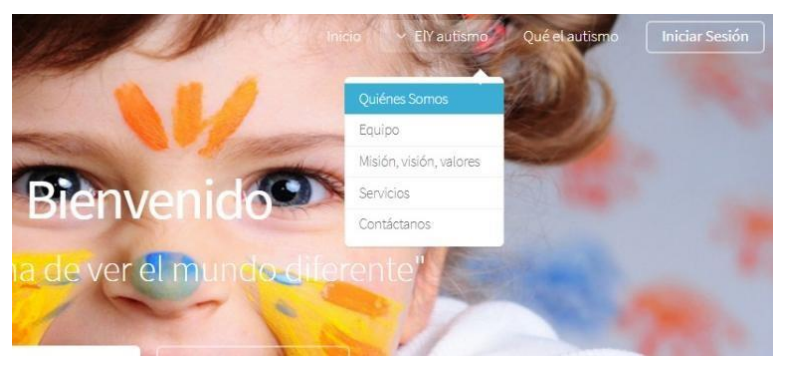

Figura 6 Juego del Rompecabezas (Elaboración Propia, 2019)

Ahora bien, en el apartado de terapias el tutor puede visualizar las fechas de las próximas terapias que tendrá su hijo, así como la hora en la que se llevará a cabo. Ver figura 7.

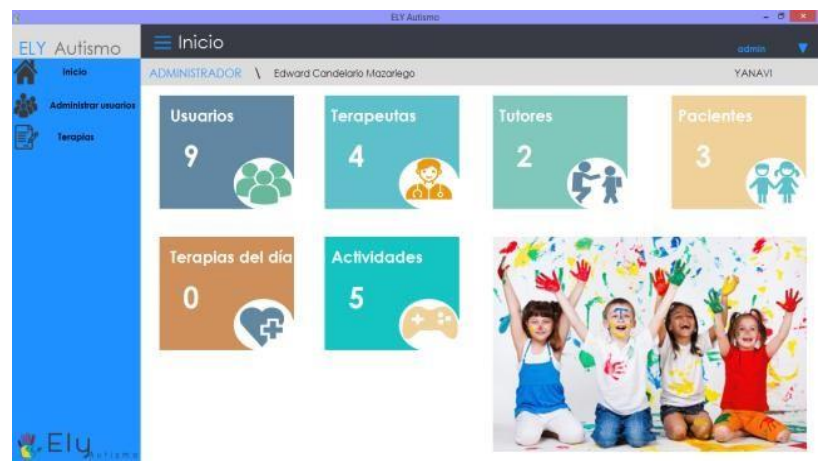

Figura 7 Juego del Rompecabezas (Elaboración Propia, 2019)

El terapeuta puede asignar las fechas de las próximas terapias que tendrá el paciente, así como la hora en la que se llevará a cabo. En la sección de resultados aparecen los resultados que ha obtenido el niño en las actividades que ha realizado graficados. Ver figura 8. 


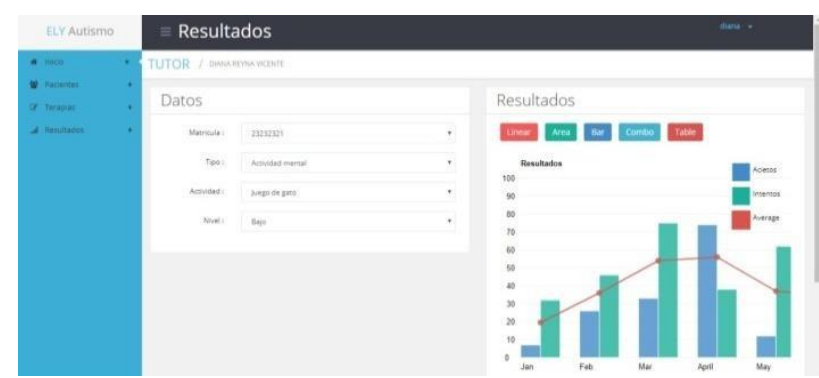

Figura 8 Juego del Rompecabezas (Elaboración Propia, 2019)

\section{Contribución}

Los resultados obtenidos en la escala valorativa de comunicación verbal la cual representa el nivel de los niños para interpretar las palabras, los signos sonoros o los auditivos. Se puede notar que hay un incremento en el desarrollo de estas habilidades después de usar el software ELY. Ver Gráfica 1.

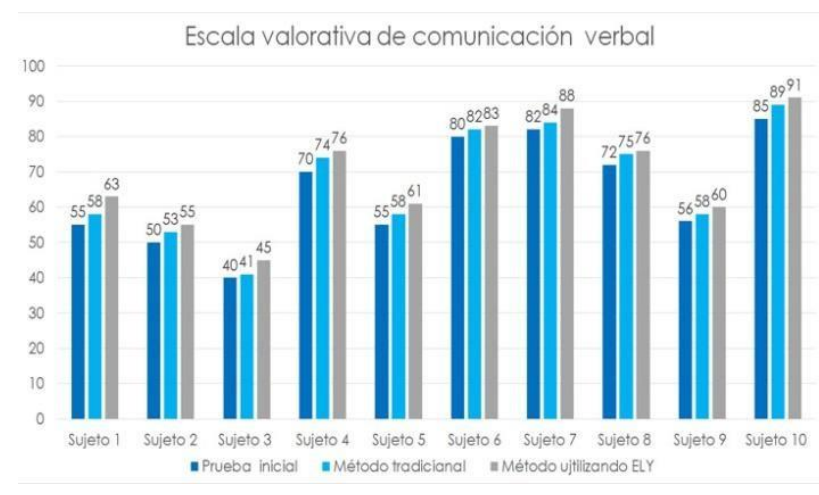

Gráfica 1 Escala valorativa de comunicación verbal (Elaboración Propia, 2019)

En la Gráfica 2 se exponen los resultados de la escala valorativa de relaciones sociales, se puede notar que los pacientes al utilizar ELY tuvieron un avance de cuatro puntos como máximo en comparación con el método tradicional que fue de tres puntos como máximo.

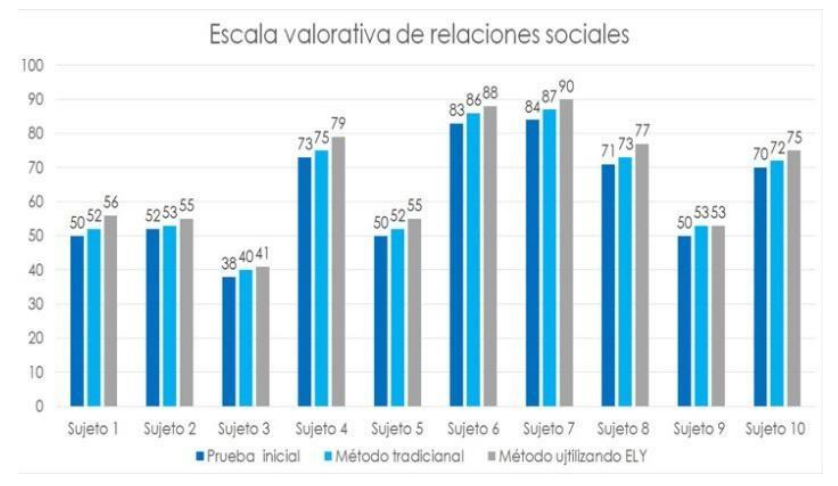

Gráfica 2 Escala valorativa de relaciones sociales (Elaboración Propia, 2019)
Las deducciones de la escala valorativa de imitación motriz donde se puede observar un incremento mayor con el uso del software ELY en comparación con el método tradicional.

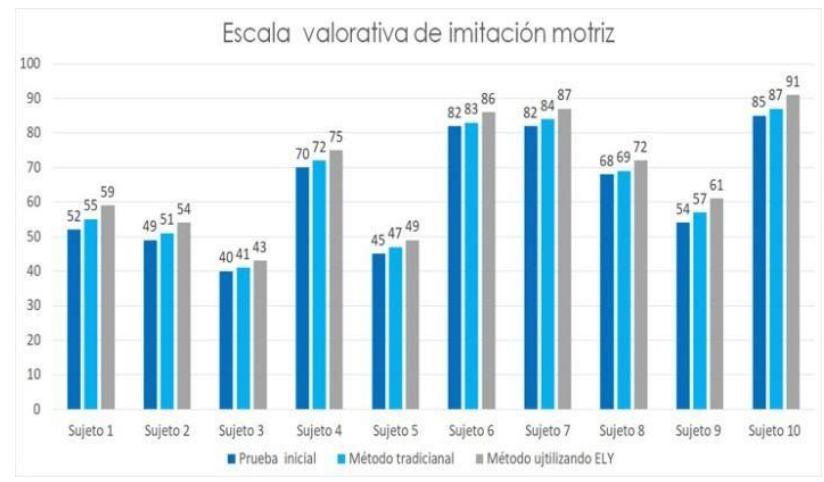

Gráfica 3 Escala valorativa de imitación motriz (Elaboración Propia, 2019)

\section{Conclusiones}

La intervención del sistema ELY como apoyo en el tratamiento de los niños participantes en la investigaciónpermitió reforzar habilidades sociales y cognitivas en menor tiempo comparado con el tratamiento tradicional lo cual afirma la hipótesis planteada inicialmente.

La inclusión en la sociedad de los niños con TEA depende hasta cierto punto de la elaboración de recursos y herramientas que implementen las TIC's, una de esas herramientas es el sistema ELY.

Actualmente se está trabajando en el desarrollo de una versión del software para utilizarse en celulares y tablets (Android) que incluya nuevas actividades considerando las opiniones y experiencias de los especialistas que trabajan y atienden a los niños autistas.

La principal meta a implementar en un futuro al proyecto es conseguir organizaciones educativas especializadas en niños con síndrome de asperger que permitan realizar pruebas contundentes en sus instalaciones.

\section{Referencias}

Acedo, M., Herrera, S., \& Traver, M. (2018). Las TIC como herramienta de apoyo para personas con Trastorno del Espectro Autista (TEA). Obtenido de https://dialnet.unirioja.es/servlet/articulo?codig $\mathrm{o}=560028$

2

GÓMEZ-MANUEL, Esbeidy, DOMÍNGUEZ-CAMPOMANES, Margarita, KATT-MORALES, Luz Alondra y GILBON-ABURTO, Antonio. Software Educativo como herramienta de apoyo para niños autistas. Revista de Arquitectura y Diseño. 2019 
American Pyschiatric Association, (2013). Diagnostic and Statistical Manual of Mental Disorders (5th revised). Washington, DC: American Pyschiatric Association. Asociación Americana de Psiquiatría (2002). Manual de diagnóstico y estadísticos de los trastornos mentales. Barcelona.

Berenguer-Forner, C, Miranda-Casas, A, Pastor- Cerezuela, G y RosellóMiranda, R. (2015). Comorbilidad del trastorno del espectro autista y el déficit de atención con hiperactividad. Estudio de revisión. Revista de neurología, 60 (Supl 1), S37-43. CABERO, J. Y GISBERT, M. (2002). Materiales formativos multimedia en la red. Guía práctica para su diseño. Sevilla, S.A.V. GONZÁlEZ CASTAÑóN, M. A. (2000).

Cornelio-Nieto, J.O. (2009). Autismo infantil y neuronas en espejo. Revista de neurología, 48 (Supl 2), S27-S29. Desconocido (2015) Las aplicaciones, el nuevo gran filón de la industria de móviles. Obtenido de http://www.expansion.com/2015/02/27/empres as/tecnolo gia/1425069142.html

Desconocido. (2013). Historia de Android: La Evolución a lo largo de sus versiones .Obtenido de http://androidzone.org/2013/05/historia-deandroid- evolucion-versiones/

González, J., y Cordero, J. (2001). Diseño de páginas web. España: Mc Graw Hill.

Humanos, H. -D. (5 de septiembre de 2017). Centro para el Control y la Prevención de Enfermedades. Obtenido de https://www.cdc.gov/spanish/

Kanner, L. (1943). Trastornos autistas del contacto afectivo. Revista Española de Discapacidad Intelectual Siglo Cero, 36.

López Gómez, S, Rivas Torres, R.M y Taboada Ares, E. A. (2009). Revisiones sobre el autismo. Revista Latinoamericana de Psicología, 41(3): 555-570.
Martínez, J., Reche, I., Martínez, C., \& Guerra, E. (2018). El desarrollo de habilidades emocionales y sociales en alumnado con trastorno del espectro autista: una investigación colaborativa en Educación Infantil y Primaria. Obtenido de https://dialnet.unirioja.es/servlet/articulo?codig $\mathrm{o}=500876$

4

Martínez-Sanchis, S. (2015). Papel de la corteza prefrontal en os problemas sensoriales en los niños con trastornos del espectro autista y su aplicación en los aspectos sociales. Revista de Neurología, 60 (Supl 1). S19-S24.

Martos. Pérez, J. (2006). Autismo, neurodesarrollo y detención temprana. Revista de Neurología, 42 (Supl 2), S99-S101.

MEDINA RIVILLA, A. (1995). Investigación en tecnología educativa, en, Tecnología educativa. Nuevas tecnologías aplicadas a la educación. RODRÍGUEZ DIÉGUEZ, J.L. Alcoy,Marfil.

MOLINA, E. y ORTEGA, J.A. (2004). Evaluando la calidad en los entornos virtuales de aprendizaje: pautas de organización, creación, legibilidad y estilo, en LatinEduca2004.com. Primer Congreso Virtual Latinoamericano de Educación a Distancia. Obtenido de http://www.ateneonline.net/datos/87_01_Ferna ndez_moli na.pdf

Ortiz M. (2 de abril de 2016). Versiones Los distintos ángulos de las noticias. Obtenido de https://www.versiones.com.mx/registraveracruz-20-mil- casos-de-autismo-apenas-seatienden-350/

Palau-Baduell, M, Salvadó-Salvadó, B, Clofent-Torrentó, M y Valls-Santasusana, A. (2012). Autismo y conectividad neuronal. Revista de neurología, 54 (Supl 1), S31-9.

PRABEESH, R. (2017) Tutoriales de diseño de aplicaciones. Obtenido de https://www.youtube.com/user/TICOONTECH NOLOGI ES/videos 
Prior, M, Eisenmajer, R, Leekam, S, Wing, L y Gould, J. (1998) Los subgrupos frente al espectro: un análisis de un grupo muestra de niños autistas. La esperanza no es un sueño: V Congreso Internacional Autismo- Europa, 1. Universidades de Melbourne y La Trobe, Australia: Canterbury U.K y Natiaonal Society for Autistic Children U.K.

Pujol, L. (1999). Los hipermedios como herramienta para facilitar el aprendizaje significativo: una perspectiva constructivista. Agenda académica. Vol.6. No.2.Caracas.Universidad Simón Bolívar

Richer, J. (1998). La detección precoz y el tratamiento de la conducta en el aspectro autista en el condado de Oxford. La esperanza no es un sueño: V Congreso Internacional Autismo-Europa, Universidades de Melbourne y La Trobe, Australia: Canterbury U.K y Natiaonal Society for Autistic Children U.K.

Riviere, A (1998). Actividad y sentido en autismo. La esperanza no es un sueño: V Congreso Internacional Autismo-Europa, 1. Universidad Autónoma de Madrid. 